\title{
Computing Viscous Flow Along a 2D Open Channel Using the Immersed Interface Method
}

\author{
Sarah Patterson ${ }^{1}$ and Anita Layton ${ }^{2}$ \\ ${ }^{1}$ Virginia Military Institute \\ ${ }^{2}$ University of Waterloo
}

May 5, 2020

\begin{abstract}
We present a numerical method for simulating 2D flow through a channel with deformable walls. The fluid is assumed to be incompressible and viscous. We consider the highly viscous regime, where fluid dynamics are described by the Stokes equations, and the less viscous regime described by the Navier-Stokes equations. The model is formulated as an immersed boundary problem, with the channel defined by compliant walls that are immersed in a larger computational fluid domain. The channel traverses through the computational domain, and the walls do not form a closed region. When the walls deviate from their equilibrium position, they exert singular forces on the underlying fluid. We compute the numerical solution to the model equations using the immersed interface method, which preserves sharp jumps in the solution and its derivatives. The immersed interface method typically requires a closed immersed interface, a condition that is not met by the present configuration. Thus, a contribution of the present work is the extension of the immersed interface method to immersed boundary problems with open interfaces. Numerical results indicate that this new method converges with second-order accuracy in both space and time, and can sharply capture discontinuities in the fluid solution.
\end{abstract}

\section{Hosted file}

ComputingViscousFlowAlong2DOpenChannel.pdf available at https://authorea.com/users/293650/ articles/421709-computing-viscous-flow-along-a-2d-open-channel-using-the-immersedinterface-method 